\title{
Computer-based digital measurement system for optical small signals
}

\author{
Lingbo Wang ${ }^{1, \mathrm{a}}$ and Fan $\mathrm{Nan}^{2}$ \\ ${ }^{1}$ College of Electronic Information Engineering, Wuhan Polytechnic, Wuhan 430070, Hubei, \\ P.R.China \\ ${ }^{2}$ School of Physics and Technology, Wuhan University, Wuhan 430072, Hubei, P.R.China \\ awlb0125@163.com
}

Keywords: boxcar integrator, lock-in amplifier, small signal detection, computer-based, LabView.

\begin{abstract}
Boxcar integrator and lock-in amplifier for the detecting of optical small signals are developed based on the virtual instrument technology of LabView. The algorithm of boxcar integrator is simple and easy-achieved. Lock-in amplifier is a powerful technique for the extracting and amplification of small signals. However, the cost of lock-in amplifier hardware is high. We develop digital boxcar integrator and lock-in amplifier in LabView platform. The performances of these two virtual measurement systems for optical small signal are exhibited and analyzed.
\end{abstract}

\section{Introduction}

In the biomedical detection, optical sensing and fundamental research, the measurement of optical small signals is a difficult work frequently encountered.. Optical small signals will be easy contaminated by noise, such as the environment light, the noise of photoelectric conversion, the noise of electric circuit, etc. The measurement system should minimize the effects of noise as far as possible.

There are many detecting technologies, for instance correlation detection and narrow-band filter, aiming small signal measurement. Lock-in amplifier is a powerful correlation detection technique for small signal detection with high signal-to-noise ratio [1,2]. However, the cost for the hardware of lock-in amplifier is very high. When the signal is a periodic signal, time domain averaging is an effective method to extract signal from complex signals with noise [3-5]. In this paper, we develop two kinds of virtual instrument based LabView platform, digital boxcar integrator and digital lock-in amplifier, for the detecting of optical small signals. The performances of these two virtual measurement systems for are exhibited and analyzed.

Modulation of Optical Signals. In optical signal measurement system, the optical signals from samples usually are very weak. Here, we realize two digital processing systems for the detection of small signals: boxcar integrator and lock-in amplifier. First of all, the optical small signals should be modulated. Figure 1 displays the using of optical chopper to modulate the signal light. The optical signals are converted to square wave by the optical chopper and then are measured by photoelectric detector, like highly sensitive avalanche photo diode (APD). The periodic signals with certain frequency are entered into conventional acquisition system undergoing amplification, filter, D/A conversion, processing and storage. Next, we mainly discuss the data processing of the complex signals of the chopped signal mixed with noise for extracting the weak effective information from sample. 


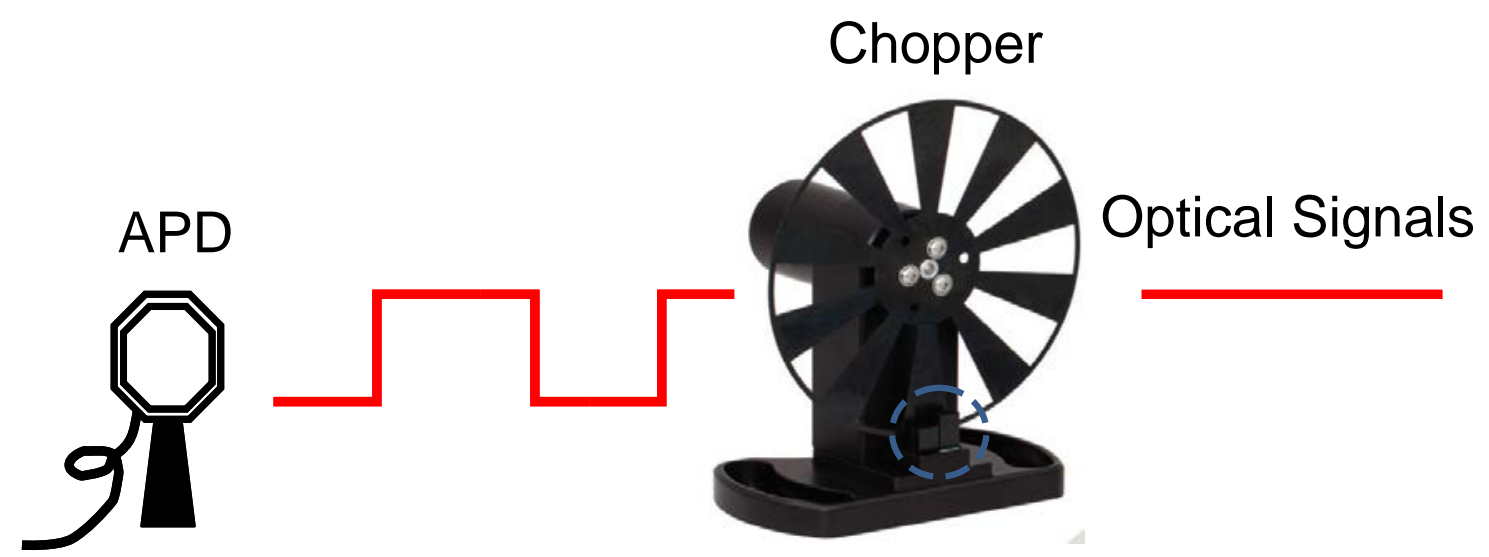

Figure 1 Schematically illustrating modulation of small signals by optical chopper.

Boxcar Integrator. We firstly exhibit the development of boxcar integrator, which is also known as boxcar average, gated integrator, referring to averaging the output of the gated integrator. The weak repeating signals are integrated to improve the signal-to-noise ratio, while the contribution of random noise is suppressed.

The noisy signal could be written as

$x(t)=s(t)+n(t)$,

where $s(t)$ is the effective periodic signal and $n(t)$ is the white Gauss noise. For the periodic signal, the sampling and integration will lead to $s_{\text {out }}=k s_{\text {in }}, k$ is the sampling times. While for the random noise, the output should follow $n_{\text {out }}=\sqrt{k} n_{\text {in }}$. Then, the output signal-to-noise ratio

$$
S N R_{\text {out }}=s_{\text {out }} / n_{\text {out }}=k s_{\text {in }} / \sqrt{k} n_{\text {in }}=\sqrt{k} s_{\text {in }} / n_{\text {in }}=\sqrt{k} S N R_{\text {in }} \text {. }
$$

The signal-to-noise ratio is improved and dependent on the sampling times.

We design the boxcar integrator in LabView, as shown in Figure 2. The simulation results are shown in Figure 3. The square wave is mixed with random noise. The noise intensity is set to 1, 3, 5, 10 , and 15 times larger than that of square wave signal, respectively. It can be seen that the square wave signals are successfully extracted from the complex input signals with noise.

The boxcar integrator is suit for extracting the complex and wide-band periodic signals as well as pulse signals. The algorithm of boxcar integrator is simple and easy-achieved. Furthermore, the sampling method, sampling frequency, integration time and other parameters could be targetedly set and optimized for signals.

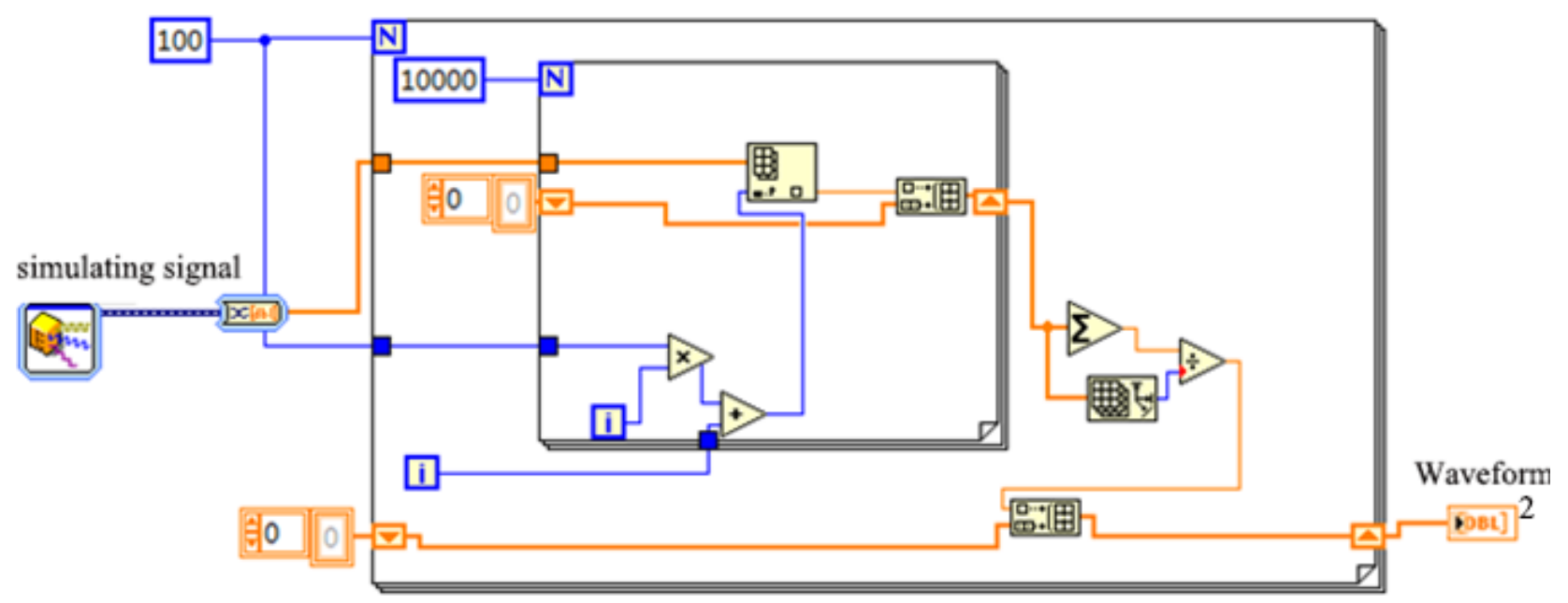

Figure 2 LabView design for boxcar integrator. 

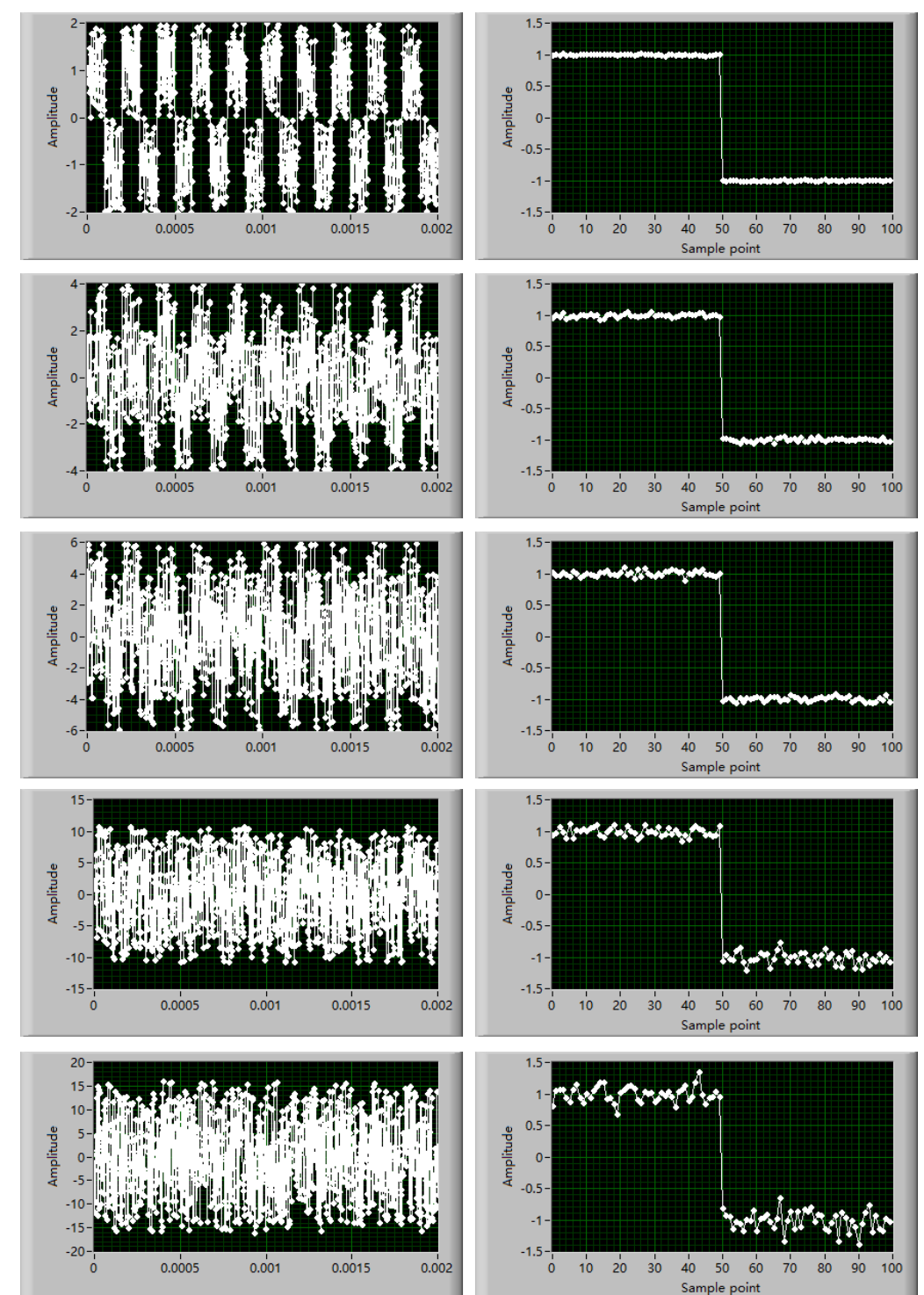

Figure 3 Small signal extracting by digital boxcar integrator. Left: the square wave is mixed with noise. Right: the extracted square wave. From top to bottom, the noise intensity is 1, 3, 5, 10, and 15 times larger than that of square wave signal, respectively.

Lock-in amplifier. Lock-in amplifier is used to measure the ultra small periodic signal through calculating the correlation of the input signal and the reference signal. The technique known as phase-sensitive detection is applied to extract the component of effective signal which has the same frequency with reference signal. While the noise signals at frequency other than the reference frequency are suppressed and rejected from the measurement.

As shown in Figure 1, the signal is modulated by the chopper with the angular frequency of $\omega_{\mathrm{s}}$. The periodic signal mixed with random noise could be written as $x(t)=s(t)+n(t)$, where $s(t)=$ $A \sin \left(\omega_{s} t+\varphi\right)$ is the effective periodic signal and $n(t)$ is the random noise. We set two reference signals having the same frequency with $s(t)$. The two reference signals have the opposite phase, which are written as $r_{\mathrm{s}}(t)=\sin \left(\omega_{\mathrm{s}} t\right)$ and $r_{\mathrm{c}}(t)=\cos \left(\omega_{\mathrm{s}} t\right)$. The signals are sampled with frequency of $\omega_{\mathrm{d}}\left(\omega_{\mathrm{d}}>>\omega_{\mathrm{s}}\right)$. The input signal, two reference signals are respectively written as $x(k)=A \sin (2 \pi k / N$ $+\varphi)+n(k), r_{\mathrm{s}}(k)=\sin (2 \pi k / N)$ and $r_{\mathrm{c}}(k)=\cos (2 \pi k / N)$, where $k=0,1, \ldots, N-1$ and $N$ is the sampling number. Then we could calculate the correlation of input signal and reference signals as

$$
R_{x r}=R_{s r}+R_{n r}=\frac{1}{N} \sum_{k=0}^{N-1} x(k) r(k)=\frac{1}{N} \sum_{k=0}^{N-1} s(k) r(k)+\frac{1}{N} \sum_{k=0}^{N-1} n(k) r(k) .
$$

$R_{\mathrm{nr}}=0$ because the noise signal is not correlated with reference signals. Then 


$$
\begin{aligned}
& R_{x r-s}=R_{s r-s}=\frac{1}{N} \sum_{k=0}^{N-1} A \sin \left(\frac{2 \pi k}{N}+\varphi\right) \sin \left(\frac{2 \pi k}{N}\right)=\frac{A}{2} \cos (\varphi)-\frac{A}{2 N} \sum_{k=0}^{N-1} \cos \left(\frac{4 \pi k}{N}+\varphi\right), \\
& R_{x r-c}=R_{s r-c}=\frac{1}{N} \sum_{k=0}^{N-1} A \sin \left(\frac{2 \pi k}{N}+\varphi\right) \sin \left(\frac{2 \pi k}{N}\right)=\frac{A}{2} \sin (\varphi)+\frac{A}{2 N} \sum_{k=0}^{N-1} \sin \left(\frac{4 \pi k}{N}+\varphi\right) .
\end{aligned}
$$

If the sampling is set for the whole function period, $R_{x r-s}=A \cos (\varphi) / 2$ and $R_{x r-c}=A \sin (\varphi) / 2$. Then we could extract the amplitude of effective periodic signal from complex input signal with random noise as

$$
A=2 \sqrt{{R_{x r-s}{ }^{2}+R_{x r-c}^{2}}^{2}} .
$$

Figure 4 shows the design of lock-in amplifier following above theory in LabView. In the simulation, the amplitude of effective periodic signal is set as $1 \mathrm{~V}$ and the noise amplitude is set 20 times larger. As the simulation results shown in Figure 5, the amplitude of effective periodic signal could be extracted by the digital lock-in amplifier.

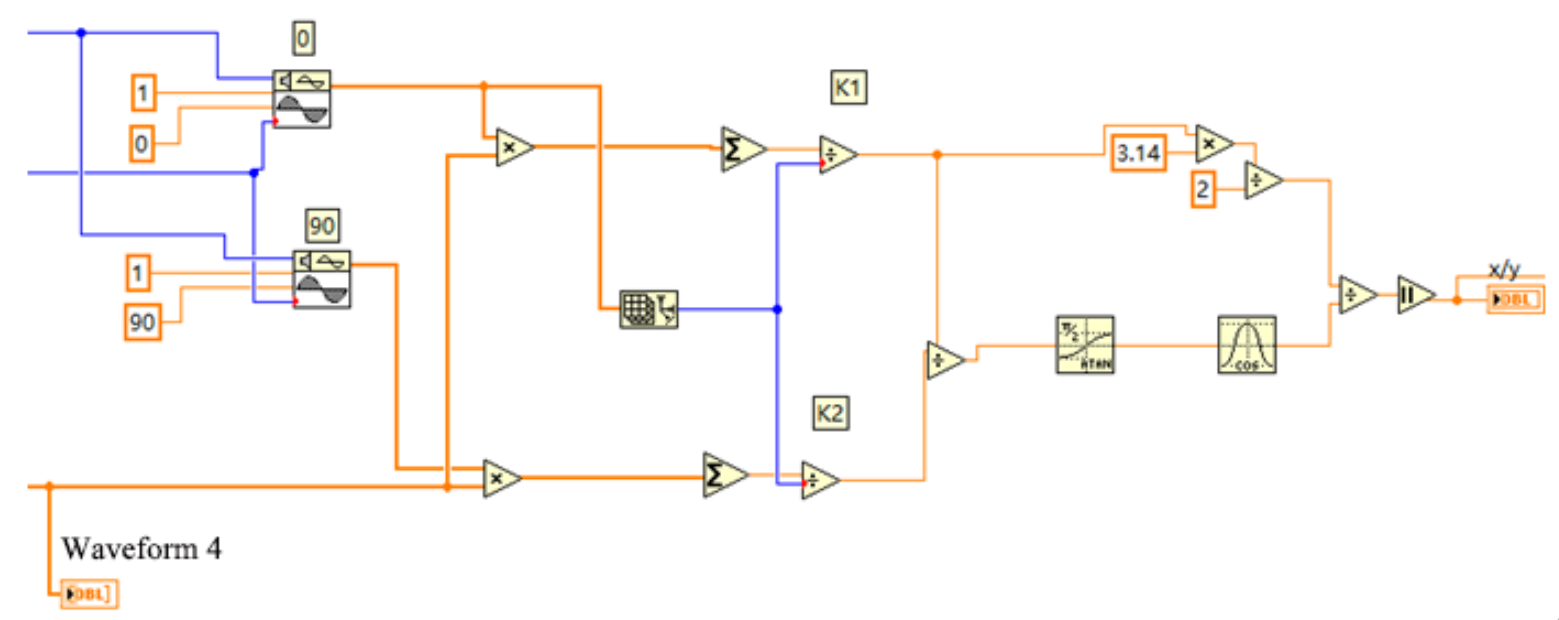

Figure 4 LabView design for lock-in amplifier.
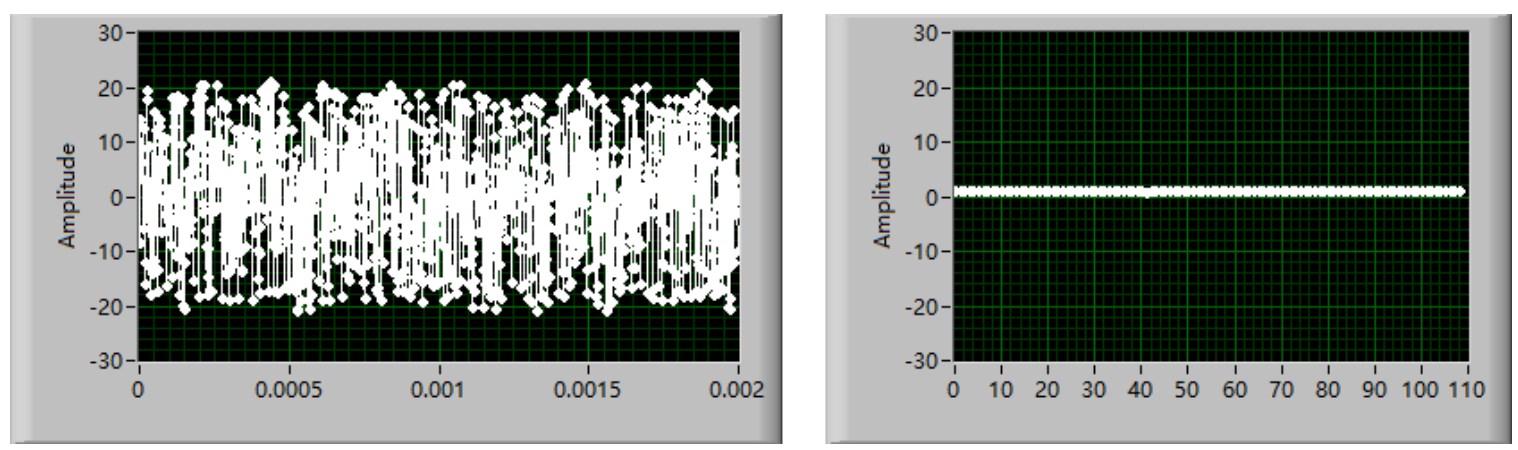

Figure 5 Small signal extracting using digital lock-in amplifier. Left: the $1 \mathrm{~V}$ signal is mixed with 20 times noise. Right: the extracted amplitude of signal, which is $1 \mathrm{~V}$ with fluctuation.

We also compare the extracting of the signal with different noise amplitudes. As shown in Figure 6, the amplitudes of effective periodic signals are extracted. The signal-to-noise of the extracted signal is decreased as the noise amplitude is increased.

The lock-in amplifier is suit for extracting the narrow-band periodic signals, which is used to detect and measure ultra small signals, down to a few nanovolts. Accurate measurements could be performed even when the small signal is obscured by the noise sources many thousands of times larger. The extracting performance of the digital lock-in amplifier exhibited here is comparable with the hardware lock-in amplifier, while the cost is very low. 


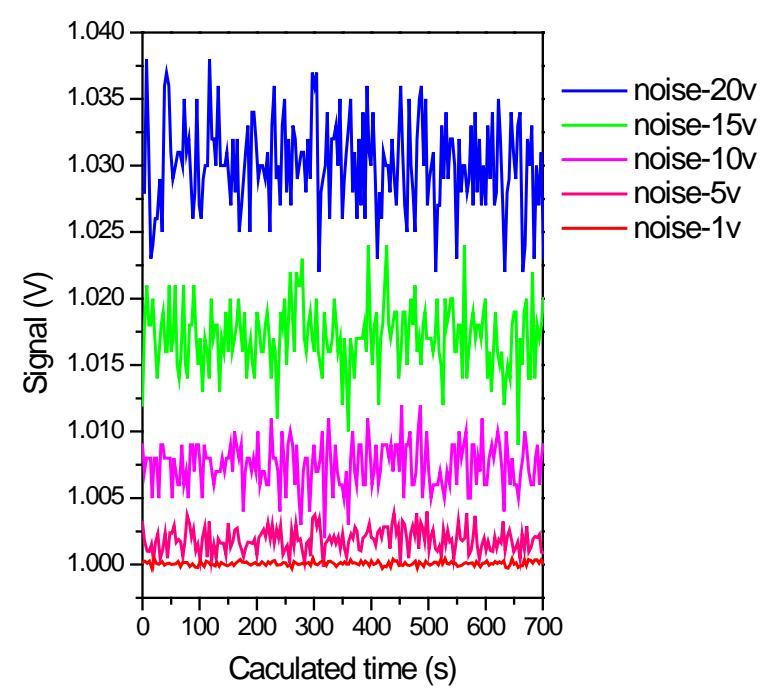

Figure 6 Comparison of small signal extracting in the presence of different noise intensity. The lines are vertically shifted for better demonstration.

\section{Summary}

In summary, we design digital boxcar integrator and digital lock-in amplifier in LabView platform. The simulation results show the periodic signals could be extracted from the complex input signals mixed with large noise. The algorithm of boxcar integrator is simple and easy-achieved. Lock-in amplifier is a powerful technique for the extracting and amplification of small signals. The computer-based digital lock-in amplifier exhibits high performance and low cost. The measurement method based on virtual instrument for optical small signals could be applied in the field of biomedical detection, optical sensing and fundamental research.

\section{References}

[1] P. Clarkson, T. J. Esward, P. M. Harris, A. A. Smith and I. M. Smith, Software simulation of a lock-in amplifier with application to the evaluation of uncertainties in real measuring systems, Measurement Science and Technology Vol. 21 (2010), p. 045106.

[2] R. Davies and G. Meuli, Development of a Digital Lock-in Amplifier for Open-path Light Scattering Measurement, The 2010 IEEE Symposium on Industrial Electronics \& Applications (ISIEA 2010).

[3] R. Suzuki, K. Umezu, H. Takuma, and F. Shimizu, Multiple sampling digital boxcar integrator, an efficient signal average, Review of Scientific Instruments Vol. 52 (1981), p. 287.

[4] Y. Wei, X. Zhu, H. Wang and G. Yang, A Weak Signals De-Noising Method Based On Virtual Instrument Technology, The Eighth International Conference on Electronic Measurement and Instruments (ICEMI 2007).

[5] Y. Yang, Periodical weak signal detection based on LabView, IEEE International Conference Neural Networks \& Signal Processing (ICNNSP 2008). 